OPEN ACCESS

Edited by:

Andrew S. Lang,

Memorial University of Newfoundland,

Canada

Reviewed by:

Steven Wilhelm,

The University of Tennessee,

Knoxville, United States

Frank O'Neill Aylward,

Virginia Tech, United States

Douwe Maat,

Royal Netherlands Institute for Sea

Research (NIOZ), Netherlands

*Correspondence:

Yaiza M. Castillo

yaiza@icm.csic.es

Specialty section: This article was submitted to Virology,

a section of the journal

Frontiers in Microbiology

Received: 04 February 2020

Accepted: 16 June 2020

Published: 17 July 2020

Citation:

Castillo YM, Sebastián M, Forn I, Grimsley N, Yau S, Moraru C and Vaqué D (2020) Visualization of Viral Infection Dynamics in a Unicellular

Eukaryote and Quantification of Viral Production Using Virus Fluorescence in situ Hybridization.

Front. Microbiol. 11:1559. doi: 10.3389/fmicb.2020.01559

\section{Visualization of Viral Infection Dynamics in a Unicellular Eukaryote and Quantification of Viral Production Using Virus Fluorescence in situ Hybridization}

\author{
Yaiza M. Castillo"*, Marta Sebastián ${ }^{1,2}$, Irene Forn ${ }^{1}$, Nigel Grimsley $^{3}$, Sheree Yau ${ }^{1,3}$, \\ Cristina Moraru ${ }^{4}$ and Dolors Vaqué ${ }^{1}$
}

'Department of Marine Biology and Oceanography, Institute of Marine Sciences (CSIC), Barcelona, Spain, 2Institute of Oceanography and Global Change (IOCAG), University of Las Palmas de Gran Canaria (ULPGC), Las Palmas de Gran Canaria, Spain, ${ }^{3}$ Integrative Biology of Marine Organisms (BIOM), Sorbonne University, CNRS, Oceanographic Observatory of Banyuls, Banyuls-sur-Mer, France, ${ }^{4}$ Department of the Biology of Geological Processes, Institute for Chemistry and Biology of the Marine Environment, Oldenburg, Germany

One of the major challenges in viral ecology is to assess the impact of viruses in controlling the abundance of specific hosts in the environment. To this end, techniques that enable the detection and quantification of virus-host interactions at the single-cell level are essential. With this goal in mind, we implemented virus fluorescence in situ hybridization (VirusFISH) using as a model the marine picoeukaryote Ostreococcus tauri and its virus Ostreococcus tauri virus 5 (OtV5). VirusFISH allowed the visualization and quantification of the proportion of infected cells during an infection cycle in experimental conditions. We were also able to quantify the abundance of free viruses released during cell lysis, discriminating OtV5 from other mid-level fluorescence phages in our non-axenic infected culture that were not easily distinguishable with flow cytometry. Our results showed that although the major lysis of the culture occurred between 24 and $48 \mathrm{~h}$ after OtV5 inoculation, some new viruses were already produced between 8 and $24 \mathrm{~h}$. With this work, we demonstrate that VirusFISH is a promising technique to study specific virus-host interactions in non-axenic cultures and establish a framework for its application in complex natural communities.

Keywords: virus fluorescence in situ hybridization, Ostreococcus tauri, Ostreococcus tauri virus 5, virus-host interactions, culture system, marine picoeukaryote

\section{INTRODUCTION}

Marine viruses have been studied during the last 3 decades mostly using microscopy (Noble and Fuhrman, 1998) and flow cytometry (FCM; Marie et al., 1999) for the enumeration and estimation of viral production. However, in the last few years, the development of high throughput sequencing techniques has considerably changed the field, and our knowledge about viral communities has exponentially increased. These new sequencing approaches provide 
information about the viral taxonomic and genomic diversity, about their biogeography and, to a certain extent, about their potential hosts (e.g., Chow et al., 2015; Labonté et al., 2015; Castillo et al., 2019). However, they do not allow the visualization of specific virus-host interactions and the monitoring of infection dynamics, which are crucial to better understand the role of viruses in shaping microbial communities and biogeochemical cycles.

Attempts to identify virus-host associations date back to the 1990s, when the role of viruses in the marine environment started to be recognized. Hennes et al. (1995) were pioneers in using fluorescently stained virus isolates to identify and enumerate their hosts in natural communities. Years after, Tadmor et al. (2011) used microfluidic digital PCR to detect specific phage-host associations in the termite gut. With this method, they managed to directly detect the phagehost association by targeting genes from both components without culturing but with no visual representation of the infection.

A few years ago, Allers et al. (2013) developed phage fluorescence in situ hybridization (phageFISH) and used it to monitor phage infections at the single-cell level in a marine podovirus-gammaproteobacterial host system. PhageFISH uses mixtures of polynucleotide probes labeled with digoxigenin to target phage genes, and a single horseradish peroxidase (HRP) labeled oligonucleotide probe to target host rRNA. The signal from the two types of probes is amplified and visualized by catalyzed reporter deposition (CARD) of fluorescently labeled tyramides. Compared to the method from Hennes et al. (1995), where the infection was forced by adding stained viruses to identify the host within natural communities, phageFISH enables the visualization of the interaction of specific virus-host pairs, because it simultaneously targets the virus and the host. More recently developed, direct-geneFISH (Barrero-Canosa et al., 2017) uses simultaneously a mixture of polynucleotide probes directly labeled with fluorochromes, to detect specific genes, and a single oligonucleotide probe, carrying multiple fluorochromes, to identify bacterial cells.

In the present work, we combined the phageFISH and direct-geneFISH techniques to develop VirusFISH with the aim of allowing (i) the identification and quantification of specific virus-unicellular eukaryote interactions at the singlecell level and (ii) the identification and quantification of free virus particles. Our procedure involves two different steps. First, a CARD-FISH step is used to detect host cells, with HRP-labeled oligonucleotide probes targeting the 18S rRNA. Then, a gene-FISH step is applied to detect viruses, using multiple polynucleotide probes labeled with fluorochromes that target specific viral genes. Since this last step can be used to detect both intracellular viruses and free viral particles, we named it VirusFISH. To visualize the host-virus interaction, VirusFISH needs to be combined with the CARD-FISH step mentioned above.

As proof of principle, we used VirusFISH to monitor viral infections in a culture of the unicellular green alga Ostreococcus tauri with the virus $O$. tauri virus 5 (OtV5).

\section{MATERIALS AND METHODS}

\section{Host-Virus System}

O. tauri, from the Mamiellophyceae class, is an important member of the eukaryotic photosynthetic picoplankton in coastal and open sea environments (Countway and Caron, 2006; Massana, 2011). It is the smallest free-living eukaryote known $(\sim 1 \mu \mathrm{m})$, capable of rapid growth in culture (Countway and Caron, 2006), and it has an extremely simplified cellular organization (Chrétiennot-Dinet et al., 1995).

In order to develop VirusFISH, we chose the O. tauri RCC4221 (Roscoff Culture Collection, NCBI accession number txid70448) - OtV5 (NCBI accession number EU304328) system. OtV5 is a member of the Phycodnaviridae family that was isolated from the Bages lagoon in 2006 (Derelle et al., 2008). It is a fast-lytic icosahedral dsDNA virus with a 186,234 bp genome and a capsid diameter of $\sim 120 \pm 30 \mathrm{~nm}$. The infection dynamics of this virus-host system has already been described (Derelle et al., 2008).

\section{OtV5 Probe Design and Synthesis}

For the detection of the OtV5 virus, we designed 11 dsDNA polynucleotide probes (300 bp each) using the software geneProber web service (http://gene-prober.icbm.de/, see Supplementary Material section for a step-by-step description of the whole procedure). These 11 probes covered a total of 3,998 bp of the OtV5 viral genome (Supplementary Table S1), offering sufficient sensitivity to detect single genes (BarreroCanosa et al., 2017), and thus single viruses. Each probe synthesis was done by obtaining the corresponding polynucleotides by PCR, and then all probes were mixed and labeled with the Alexa594 fluorochrome, following BarreroCanosa et al. (2017). The PCR was set up as follows: 10 pg of OtV5 DNA was added to a reaction mixture containing $200 \mu \mathrm{M}$ (each) deoxyribonucleoside triphosphates (Invitrogen, USA), $1 \mu \mathrm{M}$ of each primer, 1x PCR buffer (Invitrogen), and $5 \mathrm{U}$ of Taq DNA polymerase (Invitrogen). The amplification was performed in a C1000TM Thermal Cycler (Bio-Rad) with an initial denaturation step at $95^{\circ} \mathrm{C}(5 \mathrm{~min})$, followed by 30 rounds at $95^{\circ} \mathrm{C}(1 \mathrm{~min}), \mathrm{X}^{\circ} \mathrm{C}(30 \mathrm{~s})$, and $72^{\circ} \mathrm{C}(30 \mathrm{~s})$, and a final extension at $72^{\circ} \mathrm{C}(10 \mathrm{~min}) . \mathrm{X}$ value corresponds to the optimal annealing temperature for each of the primers determined after performing gradient PCRs. All OtV5 primers had an optimal annealing temperature of $62.5^{\circ} \mathrm{C}$, with the exception of primers $\# 3$ and $\# 5$ that had an annealing temperature of $65.5^{\circ} \mathrm{C}$. For each polynucleotide, several PCRs were done to obtain a minimum of $400 \mu \mathrm{l}$ PCR volume. This volume was purified on a single purification column using the QIAquick PCR purification kit (Qiagen, Germany, cat.no. 28106) and resuspended in a TE solution $(5 \mathrm{mM}$ Tris- $\mathrm{HCl}$ and $1 \mathrm{mM}$ EDTA, pH 8.0). The polynucleotide length was checked by agarose gel electrophoresis, and the concentration was measured spectrophotometrically using a NanoDrop 1000 (Fisher Thermo Scientific). Then, all 11 polynucleotides were mixed equimolarly to yield a total of $1 \mu \mathrm{g}$ DNA in $10 \mu \mathrm{l}$ TE. Later, the probe mixture was heated to $95^{\circ} \mathrm{C}$ for $5 \mathrm{~min}$ to denature it and then incubated for $30 \mathrm{~min}$ at $80^{\circ} \mathrm{C}$ with $10 \mu \mathrm{l}$ of the Alexa594 
dye for the fluorescent labeling (Ulysis ${ }^{\mathrm{TM}}$ Alexa Fluor ${ }^{\circledR} 594$ Nucleic Acid Labeling Kit, Thermofisher, MA, USA, cat.no: U21654). The unbound Alexa594 was removed using chromatography columns (Micro Bio-spin chromatography columns P-30, Bio-Rad, California, USA, cat.no. 732-6202). The concentration of the probe mixture and the labeling efficiency with the Alexa594 were determined spectrophotometrically using a NanoDrop 1000 with the multiarray option and N-50. For a successful detection of the virus, we observed that the average labeling efficiency should be at least six Alexa594 dyes per probe (see further details in the step-by-step protocol in the Supplementary Material section). Fluorescent probes were stored at $-20^{\circ} \mathrm{C}$ until use.

\section{Experimental Viral Infection of O. tauri}

The host strain O. tauri RCC4221 was grown in $60 \mathrm{ml}$ of L1 medium (Guillard and Hargraves, 1993) in aerated flasks (Sarstedt) and incubated at $21.5^{\circ} \mathrm{C}\left( \pm 0.5^{\circ} \mathrm{C}\right)$ with white light $\sim 100 \mu \mathrm{E}$ and a 10:14 h photoperiod (light:darkness), until stationary phase

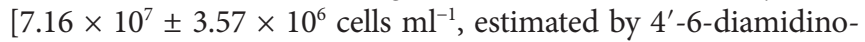
2-phenylindole (DAPI) counts (Porter and Feig, 1980), for better detection of individual cells]. Triplicate $O$. tauri cultures $(20 \mathrm{ml})$ were infected at $12 \mathrm{PM}$ with $1 \mathrm{ml}$ of OtV5 inoculum $\left(1.3 \times 10^{7} \pm 4.3 \times 10^{6}\right.$ viruses $\mathrm{ml}^{-1}$, estimated by plaque-forming units), resulting in a 0.01 multiplicity of infection (MOI). Non-infected triplicate $O$. tauri cultures (inoculated with $1 \mathrm{ml}$ of L1 medium) were used as control. After OtV5 inoculation, samples $(900 \mu \mathrm{l})$ were taken over 3 days at times $0,8,24,48$, and $72 \mathrm{~h}$, and fixed with $100 \mu \mathrm{l}$ of freshly filtered formaldehyde (3.7\% final concentration) for $15 \mathrm{~min}$ at room temperature. Then, $500 \mu$ l of fixed sample $+5 \mathrm{ml} 30 \mathrm{KDa}$ filtered sea water was filtered through $25 \mathrm{~mm} 0.2 \mu \mathrm{m}$ pore size polycarbonate white filters (Merck ${ }^{\mathrm{TM}}$ GTTP02500) to retain cells (dilution with $30 \mathrm{KDa}$ filtered sea water helps to have a homogenous distribution of cells on the filter during the filtration process without altering the sample composition). The $0.2 \mu \mathrm{m}$ pore size polycarbonate filters were embedded in $0.1 \%\left(\mathrm{w} \mathrm{v}^{-1}\right)$ low gelling point agarose to prevent cell loss, and treated for $1 \mathrm{~h}$ with $96 \%$ ethanol and $1 \mathrm{~h}$ with pure methanol, to remove cellular pigments that can interfere with the CARD-FISH signal (Supplementary Figure S1), and $10 \mathrm{~min}$ with $\mathrm{HCl}$ to inactivate endogenous peroxidases (Pavlekovic et al., 2009). All filters were kept at $-20^{\circ} \mathrm{C}$ until hybridization. For the detection of free viruses, the sample was filtered through a $25 \mathrm{~mm} 0.2 \mu \mathrm{m}$ pore size syringe filter to remove cell debris, $500 \mu \mathrm{l}$ subsamples were fixed with formaldehyde (3.7\% final concentration) for $15 \mathrm{~min}$ at room temperature, and viral particles were collected onto $25 \mathrm{~mm} 0.02 \mu \mathrm{m}$ pore size anodisc filters (Whatman $\left.{ }^{\circledR}\right)$.

\section{Detection of 0 . tauri Cells Using $18 S$ rRNA Targeted CARD-FISH}

O. tauri cells were labeled using Catalyzed Reporter Deposition-FISH (CARD)-FISH following Pernice et al. (2015), with the 18S rRNA Ostreococcus spp. specific probe OSTREO01 (Not et al., 2004). Briefly, the hybridization was carried out by covering filter pieces with $20 \mu$ l of hybridization buffer
(HB) containing $40 \%$ deionized formamide and incubating at $35^{\circ} \mathrm{C}$ overnight (see Supplementary Material section for details on the $\mathrm{HB}$ composition). After two successive washing steps of $10 \mathrm{~min}$ at $37^{\circ} \mathrm{C}$ in a washing buffer, and a equilibration in phosphate-buffered saline for $15 \mathrm{~min}$ at room temperature (Cabello et al., 2016), the signal was amplified for $1 \mathrm{~h}$ at $46^{\circ} \mathrm{C}$ with Alexa488-labeled tyramide. Filters were then placed in phosphate-buffered saline twice for $10 \mathrm{~min}$, rinsed with MilliQ water and air-dried.

\section{Detection of Intracellular and Free OtV5 Viruses}

OtV5 viruses were labeled using a modified version of the direct-geneFISH protocol (Barrero-Canosa et al., 2017). OtV5 associated to Ostreococcus cells were visualized on $0.2 \mu \mathrm{m}$ pore size filters that had been previously hybridized with the Ostreococcus CARD-FISH probes. Free OtV5 particles $(\sim 120 \mathrm{~nm}$ in diameter) produced during the experiment were monitored on $0.02 \mu \mathrm{m}$ pore size filters. The hybridization was done by covering the filter pieces with $25 \mu$ of $40 \%$ formamide hybridization buffer (see composition in the step-by-step protocol in the Supplementary Material section) containing the OtV5 probes and incubating first for $40 \mathrm{~min}$ at $85^{\circ} \mathrm{C}$, and then for $2 \mathrm{~h}$ at $46^{\circ} \mathrm{C}$. The volume of probe mixture labeled with Alexa594 to add to the HB was calculated based on the following formula, according to Barrero-Canosa et al. (2017):

$(25 \mu l \mathrm{HB} \cdot$ number of filters $)$.

$\left(\frac{62 \mathrm{pg}}{\mu l}\right.$ final probe concentration $\bullet$ number of total probes $) /$

Viral probe concentration $\left(\frac{\mathrm{ng}}{\mu l}\right) \cdot 1000=\mu l$ probe mixture

The formula above assumes that the volume of $\mathrm{HB}$ for each filter portion is $25 \mu \mathrm{l}$ and $62 \mathrm{pg} \cdot \mu^{-1}$ is the desired final concentration for each polynucleotide, according to Barrero-Canosa et al. (2017).

Finally, samples were washed at $48^{\circ} \mathrm{C}$ for 15 min with gentle shaking in a washing buffer (see composition in the step-by-step protocol in the Supplementary Material section), rinsed with MilliQ water and air-dried.

\section{Sample Mounting, Visualization, and Image Analysis}

The CARD-FISH signal for this tiny picoeukaryote is not very strong, because it is limited to its small cytoplasm. Thus, after hybridization, the $0.2 \mu \mathrm{m}$ filters were counterstained with DAPI at $0.5 \mu \mathrm{g} \mathrm{ml}^{-1}$ to facilitate the counting of $O$. tauri cells that appear clustered. Filters were then mounted in an antifading reagent (77\% glycerol, 15\% VECTASHIELD, and 8\% 20x PBS; Cabello et al., 2016). Images were manually acquired using a Zeiss Axio Imager Z2m epifluorescence microscope (Carl Zeiss, Germany) connected to a Zeiss camera (AxioCamHR, Carl Zeiss MicroImaging, S.L., Barcelona, Spain) at 1000x magnification through the AxioVision 4.8 software. The DAPI signal from $O$. tauri was observed with the specific UV filter 
set (370/40 nm excitation, 425/46 emission, and FT 395 beam splitter), while the $18 \mathrm{~S}$ rRNA CARD-FISH signal from O. tauri was observed using a filter set specific for Alexa488 (475/30 nm excitation, 527/54 BP emission, and FT 495 beam splitter). OtV5 was observed using a filter set specific for Alexa594 (585/35 nm excitation, 615 LP emission, and FT 570 beam splitter). All pictures were taken using the same intensities and exposure times $(400 \mathrm{~ms}$ for the $O$. tauri and $1 \mathrm{~s}$ for the virus detection).

Total free viruses (i.e., both OtV5 and phages present in the non-axenic culture), collected on the $0.02 \mu \mathrm{m}$ pore size anodisc filters, were counterstained with SYBR Gold (SYBR ${ }^{\mathrm{TM}}$ Gold solution, Invitrogen) at $2 \mathrm{x}$ final concentration for $12 \mathrm{~min}$ and then rinsed abundantly with MilliQ water to remove excess stain. Filters were finally mounted on slides with an antifading mounting solution (CitiFluor ${ }^{\mathrm{TM}}$ Glycerol-PBS Solution $\mathrm{AF} 1$ ). Images were acquired on the same Zeiss microscope and camera at $1000 \mathrm{x}$ magnification. OtV5 were observed by epifluorescence microscopy under 585/35 nm excitation, 615 LP emission wave-lengths, and FT 570 beam splitter, using $1 \mathrm{~s}$ of exposure time, and total viruses (OtV5 and phages) under 475/30 nm excitation, 527/54 BP emission wave-lengths, and FT 495 beam splitter, using $50 \mathrm{~ms}$ of exposure time. Due to the long exposure times needed to visualize the OtV5 viruses there was some non-specific Alexa594 signal (red color) in the micrographs, but co-localization with SYBR Gold (green color) resulted in a drastic reduction of this unspecificity (Supplementary Figure S2). Thus, only the VirusFISH red signal that overlapped with a SYBR Gold green fluorescence signal, was considered a true OtV5 particle. All pictures were taken using the same intensities and exposure times mentioned above. Image analysis for free virus detection was done using the software ACMEtool 3 (July 2014; M Zeder, Technobiology $\mathrm{GmbH}$, Buchrain, Switzerland).

During the image analysis, we observed that a fraction of the OtV5 virions released from the cells during lysis was trapped on an extracellular organic matrix surrounding cell debris (here referred to as viral clouds, Figure 1; Supplementary Figure S3) and retained on the $0.2 \mu \mathrm{m}$ filters. Thus, for 48 and $72 \mathrm{~h}$, when most cells were lysed, we also calculated the abundance of OtV5 retained on the $0.2 \mu \mathrm{m}$ filters. To this end, for those timepoints, 10 images of each of the triplicate cultures were analyzed using AcmeTool 3, and the area of the viral clouds around the cells was measured. The number of virus trapped in the organic matrix was obtained by dividing the total area of viral clouds in the 10 images by the average area of an OtV5 virus, obtained from the $0.02 \mu \mathrm{m}$ filters (at $48 \mathrm{~h}, n=2,432$ viral clouds areas; at $72 \mathrm{~h}, n=307$ viral clouds areas; and free OtV5, $n=30,000$ OtV5 particles areas). The total OtV5 production at 48 and $72 \mathrm{~h}$, in each of the triplicate cultures, was estimated as the sum of the free virus abundance on the $0.02 \mu \mathrm{m}$ filters plus the viral abundance retained on the $0.2 \mu \mathrm{m}$ filters. Despite this effort, we acknowledge that viral production calculated this way could be underestimated, as it is possible that some viral particles on the $0.2 \mu \mathrm{m}$ filters may be hidden behind others and not displayed on a single layer.

\section{Comparison of OtV5 Counts With VirusFISH and Flow Cytometry}

Since flow cytometry is usually used to quantify viral production during infection experiments, we compared OtV5 counts obtained with flow cytometry and VirusFISH. Triplicate $500 \mu \mathrm{l}$ samples were taken from a healthy and an infected $O$. tauri culture $96 \mathrm{~h}$ post OtV5 inoculation. Samples were filtered through a $25 \mathrm{~mm} 0.2 \mu \mathrm{m}$ pore size syringe filter to remove cells and cell debris. For VirusFISH, $500 \mu \mathrm{l}$ of the $0.2 \mu \mathrm{m}$ filtered subsamples were fixed with formaldehyde $(3.7 \%$ final concentration) for $15 \mathrm{~min}$ at room temperature, and the viral particles were collected onto $25 \mathrm{~mm} 0.02 \mu \mathrm{m}$ pore size anodisc filters $\left(\right.$ Whatman ${ }^{\circledR}$ ) and kept at $-20^{\circ} \mathrm{C}$. Later, the VirusFISH protocol was applied as explained above.

For the flow cytometry counts, the $0.2 \mu \mathrm{m}$ filtered samples were fixed with glutaraldehyde ( $0.5 \%$ final concentration) for $30 \mathrm{~min}$ at $4^{\circ} \mathrm{C}$ and counted in a FACSCalibur (Becton \& Dickinson) flow cytometer following Brussaard (2004). Briefly, samples were diluted 1/1000 from the infected culture, and 1/100 from the control, using TE-buffer (10:1 mM Tris:EDTA). Then, samples were stained with SYBR Green I ( $5 \mu \mathrm{l}$ in $500 \mu \mathrm{l}$ diluted sample), incubated at $80^{\circ} \mathrm{C}$ for $10 \mathrm{~min}$, attemperated $5 \mathrm{~min}$ in the dark, and ran at a medium flow speed with a flow rate of $64 \mu \mathrm{l} \mathrm{min}{ }^{-1}$. Counts were made for $60 \mathrm{~s}$ using the following settings: SSC 625, FL1 530. In the cytograms low-level, mid-level, and high-level fluorescence viruses were distinguished as in Brussaard et al. (2010).

\section{Viral Morphologic Characterization by Transmission Electron Microscopy}

The viral lysate of $O$. tauri was prepared for transmission electron microscopy (TEM) at the Unitat de Criomicroscopia Electrònica (Centres Científics i Tecnològics, Universitat de Barcelona). For this, $6 \mu \mathrm{l}$ of the viral lysate was placed onto a parafilm with a fresh glow-discharged coated carbon grid on them for $1 \mathrm{~min}$. The adsorbed viruses in the grid were negatively stained by adding five drops of uranyl acetate solution (2\%, final conc) for $10 \mathrm{~s}$. Excess stain was drawn off with filter paper and the grid was air-dried. The grids were observed in a Jeol 1010 (Jeol, Japan) transmission electron microscope operating at $80 \mathrm{kv}$ equipped with a CCD camera SIS Megaview III and AnalySIS software.

\section{RESULTS}

\section{The OtV5 - O. tauri Infection Dynamics as Revealed by VirusFISH}

A non-axenic culture of $O$. tauri was infected with the virus OtV5, at a MOI of 0.01 and an uninfected culture was grown in parallel, as a control (Figure 2A). Using 18S CARD-FISH combined with VirusFISH, the two cultures were followed for $72 \mathrm{~h}$, quantifying (i) the absolute abundance of $O$. tauri cells and (ii) the relative and absolute abundance of infected $O$. tauri cells. The infected culture experienced a dramatic decrease in cell density, going from $4.8 \cdot 10^{7}$ to $4.1 \cdot 10^{5}$ cells $\mathrm{ml}^{-1}$ in $48 \mathrm{~h}$ 


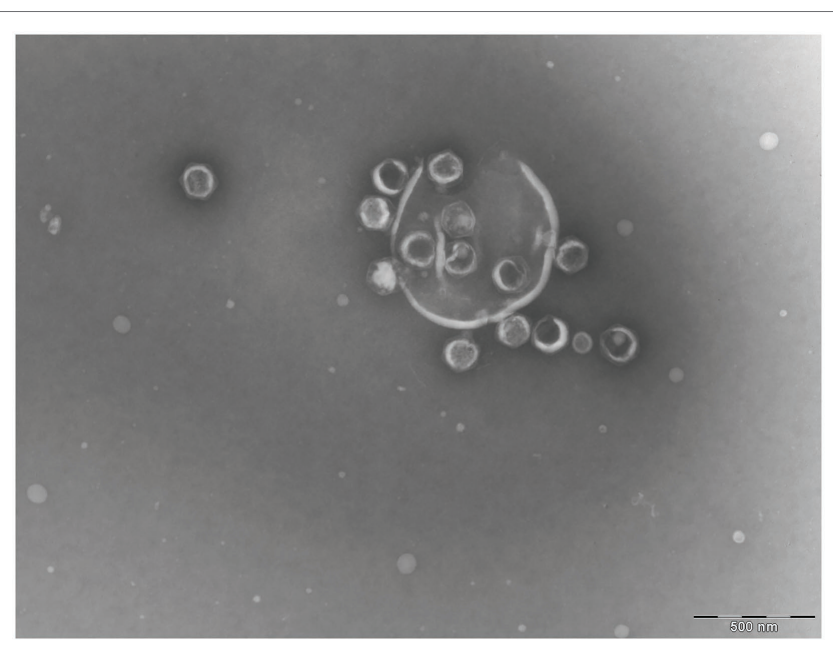

FIGURE 1 | Transmission electronic microscope image of a lysed Ostreococcus tauri cell. Released Ostreococcus tauri virus 5 (OtV5) virions are observed surrounding cell debris.

(Figures 2B, 3; Supplementary Figure S4). At $72 \mathrm{~h}$, almost no $O$. tauri cells were detected (Figure 2B; Supplementary Figure S4), consistent with the clearing of the infected culture (Figure 2A). The same infection dynamics were observed in three previous O. tauri- OtV5 experiments (data not shown). The absence of any red signal (i.e., fluorescence of the OtV5 probes) in the control treatment (Supplementary Figure S5) confirms the lack of false positives (i.e., non-specific binding of the OtV5 probes to host cells) during the hybridization.

At the MOI used, rapid adsorption of all the viral particles added would theoretically result in $1 \%$ of infected cells (considering "infected" cells those with either viruses adsorbed or actual infections). However, despite infected cells being visible as early as $0.4 \mathrm{~h}$, their abundance was very low at both 0.4 and $8 \mathrm{~h}$ (0.02 and $0.2 \%$, respectively), suggesting that not all viral particles had yet been adsorbed (Figure 4). Nevertheless, the fact that at $24 \mathrm{~h}$, we found $16 \%$ of the population infected implies that between 8 and 24 h there had been some production of viruses that had gone on to infect more cells in the culture. Later, at $48 \mathrm{~h}$, the abundance of cells decreased by two orders of magnitude and $60 \%$ of the remaining cells were infected (Figures 3, 4). In contrast, the abundance of $O$. tauri cells in the control cultures remained relatively constant along the experiment and, as expected, no infected cells were observed (Figure 2B; Supplementary Figure S5).

\section{Dynamics and Abundances of Free OtV5 Particles}

We also used VirusFISH for the detection and quantification of free OtV5 particles produced during the infection and lysis of $O$. tauri. Production of free viruses during infection experiments is often quantified with flow cytometry (e.g., Marie et al., 1999; Derelle et al., 2008). However, in our comparison of counts between VirusFISH and FCM, we observed that in late stages of infection of our non-axenic culture, an abundant population of mid-level fluorescence viruses, presumably phages, appeared in the cytogram just below the high-level fluorescence viruses, presumably OtV5 (Figure 5), but without a clear differentiation of the two close populations. OtV5 counts estimated by VirusFISH on the $0.02 \mu \mathrm{m}$ anodisc filters and putative OtV5 particles estimated by FCM were in the same order of magnitude but slightly higher by FCM $\left(7.4 \times 10^{8}\right.$ vs. $4.5 \times 10^{8}$ virus $\mathrm{ml}^{-1}$, Figure 5$)$ perhaps due to a certain overlap with the population of mid-level fluorescence phages. For this reason, we decided to use VirusFISH to quantify the production of OtV5 and also demonstrate the validity of this technique to visualize a desired virus within complex viral communities.

As mentioned above, SYBR Gold was used to counterstain the VirusFISH, which helped both to discriminate true OtV5 viruses from unspecific OtV5 probe signals (see Supplementary Figure S2) and from other phages (Figure 5B; Supplementary Figure S6). Only the VirusFISH red signal that overlapped with a SYBR Gold green fluorescence signal (yellowish particles in Figure 5B) was considered a true OtV5 particle. The number of free OtV5 viruses collected on the $0.02 \mu \mathrm{m}$ filter when the major lysis occurred $(48 \mathrm{~h}$ postinfection) was much lower than expected, representing only around $20 \%$ of total viruses (i.e., OtV5 and phages, Figure $6 \mathbf{A}$ ). We realized that, after cell lysis, the organic matrix released from the cells trapped most OtV5 particles on the $0.2 \mu \mathrm{m}$ filters (Supplementary Figure S3). We, therefore, summed together the free OtV5 particles detected on the $0.02 \mu \mathrm{m}$ filters and the OtV5 estimated within the viral clouds around the cells (see Materials and Methods section for details) and obtained that the OtV5 particles produced represented around $75 \%$ of total viruses after the major cell lysis occurred (Figure 6B). Thus, we used this approach to estimate the OtV5 produced at 48 and $72 \mathrm{~h}$ post-infection. Since there was no visible cell lysis from 0 to $24 \mathrm{~h}$, at those time points we only considered the free OtV5 viruses detected on the $0.02 \mu \mathrm{m}$ filters.

Our results showed that before $24 \mathrm{~h}$, some cell lysis had already occurred, as indicated by the slight, but detectable increase in OtV5 free particles at $24 \mathrm{~h}$ (Figures 6, 7). This agrees with the detection of $16 \%$ infected $O$. tauri cells at $24 \mathrm{~h}$, which implies that at the MOI used some viral production had already taken place, as explained above. A drastic increase in viral abundance was observed after $24 \mathrm{~h}$ (Figure 7; Supplementary Figure S7), corresponding with the time the majority of cells was lysed. At $48 \mathrm{~h}$, the number of free viruses reached a plateau, likely because most viral production had already occurred. Very low numbers of OtV5 viruses were detected in the control flasks (Figure 7; Supplementary Figure S7), likely false positives due to the long exposure time needed to acquire the images for the viral detection (Supplementary Figure S2). Yet, these false OtV5 counts were constant over the infection cycle, representing on average $0.5 \%$ $( \pm 0.1 \%)$ of total viruses (Figure 7$)$. The fraction of OtV5 within the total viral community in the infection flasks ranged from $0.9 \%( \pm 0.2 \%)$ at $0 \mathrm{~h}$ when viruses were inoculated to $72.1 \%( \pm 5.6 \%)$ at $48 \mathrm{~h}$ when almost all $O$. tauri cells were lysed (Figures 3, 7). 
A

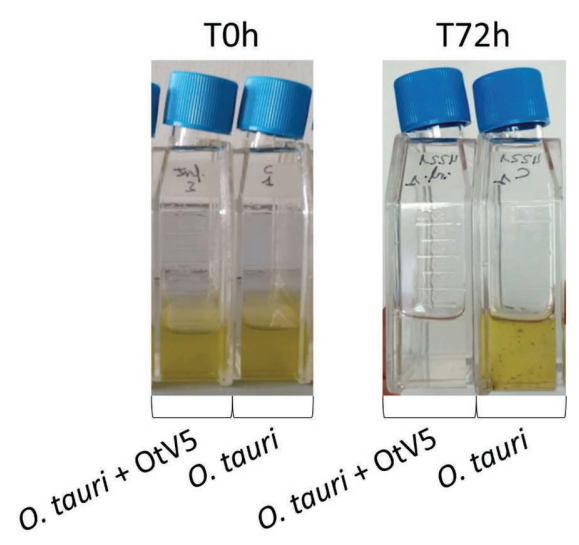

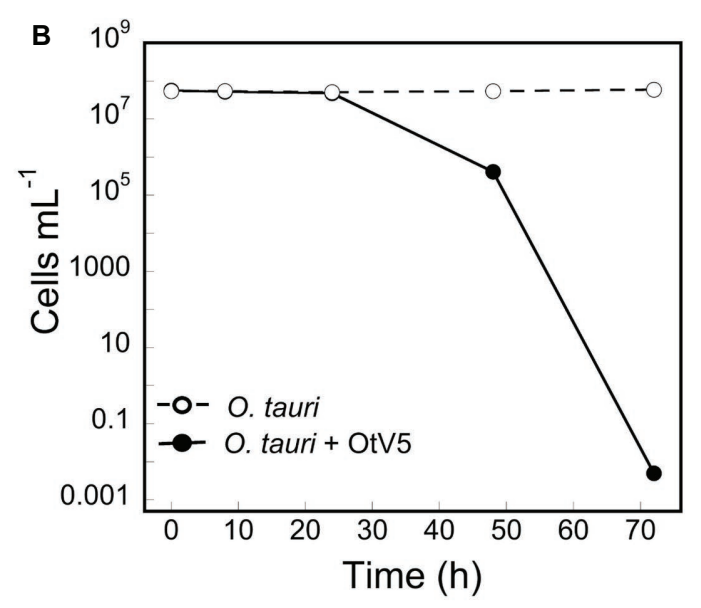

FIGURE 2 | Dynamics of the infection of O. tauri with OtV5. (A) Infection and control culture flasks at time 0 and 72 h. (B) 0 . tauri cell abundances (average \pm standard error) detected by catalyzed reporter deposition fluorescence in situ hybridization and counted by epifluorescence microscopy in both the infected (solid circles) and the control (empty circles) triplicate cultures.

\section{O. tauri signal}
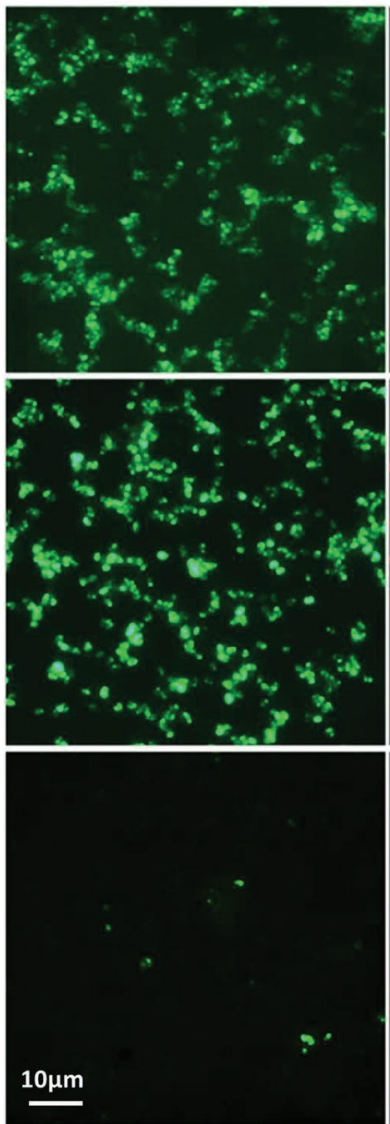

OtV5 signal
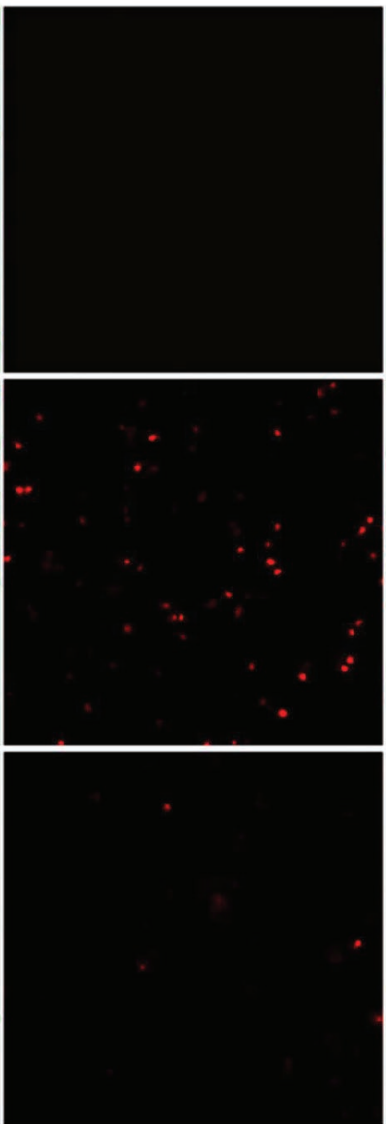

\section{Overlay}

o. tauri-OtV5

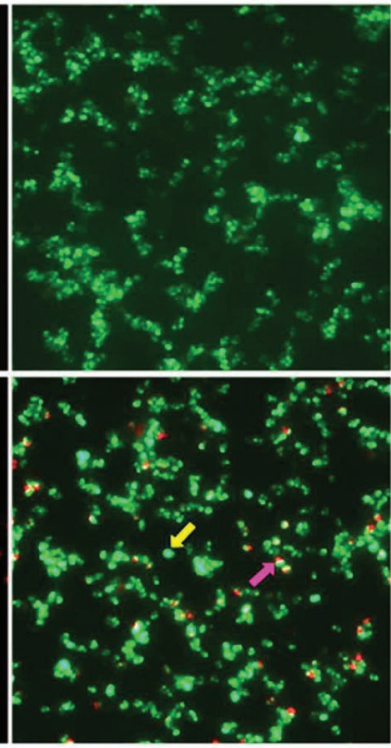

$\mathrm{T} 8 \mathrm{~h}$

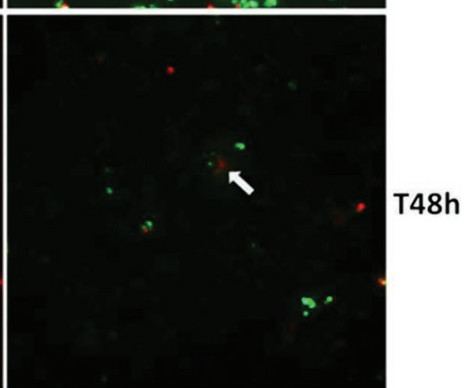

FIGURE 3 | Micrographs of the evolution of the infection from time 8 to 48 h. Left: O. tauri only. Centre: OtV5 only. Right column: overlay of O. tauri host cells in green (Alexa488) and virus in red (Alexa594). Yellow arrow: non-infected $O$. tauri, pink arrow: infected $O$ tauri, gray arrow: cloud of viruses retained on the filter by the organic matter released during the lysis. See supplementary Figures for a complete temporal overview of both the infection and the control cultures. 


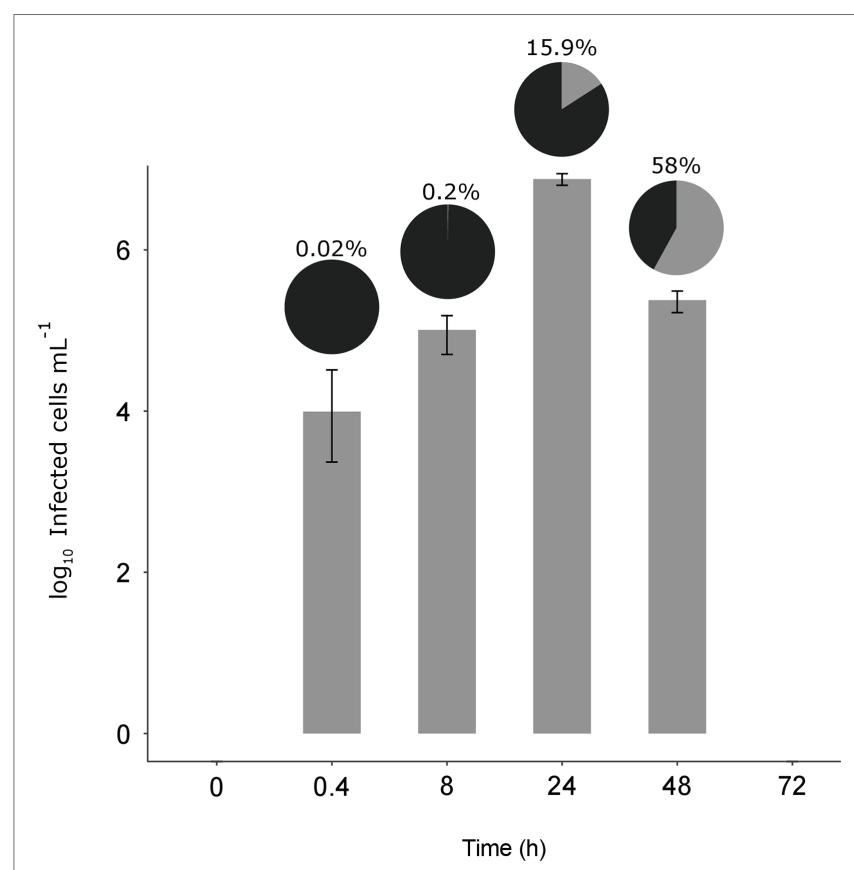

FIGURE 4 | Dynamics of the infected cells. Bar plot shows the number of infected $O$. tauri at each time ( \pm standard error of the three replicates). Pie charts on top of each bar show the percentage of infected cells (gray) with respect to the total $O$. tauri abundance.

\section{DISCUSSION}

Several studies have dealt with the virus-host relationships of the four clades of Ostreococcus spp. (O. tauri, O. lucimarinus, O. mediterraneus, and clade B; Guillou et al., 2004), and our knowledge on these systems is continuously expanding (Weynberg et al., 2017). From these studies, only a few focused on the infection dynamics (e.g., Derelle et al., 2008; Heath and Collins, 2016). Most of the work has been directed toward understanding the virus-host interaction at the molecular level (e.g., Derelle et al., 2008; Weynberg et al., 2011; Clerissi et al., 2012), unveiling, for instance, interesting information on the host resistance mechanisms to viruses (Thomas, 2011; Heath and Collins, 2016; Yau et al., 2016). However, to understand the impact of viruses on the ecology of Ostreococcus spp., it is crucial to develop techniques that enable monitoring the host-virus interactions at the single cell level, with the ultimate goal to apply them in complex natural communities. We designed probes to detect OtV5, but the alignment of the probes with other Prasinovirus genomes showed that they can potentially label all 11 genome-sequenced Ostreococcus spp. viruses (Supplementary Tables S2, S3), except OtV6, which is evolutionarily distinct (Monier et al., 2017). Thus, our technique may help fostering our knowledge on the role of viruses in the control of the abundance of the cosmopolitan Ostreococcus spp.

Unlike flow cytometry measurements and plaque-forming unit assays, which only give absolute cell and virus counts, VirusFISH allowed to follow the whole process of infection and shed light on what was happening previous to culture clearance, unveiling that infection was faster than what it could be inferred from only cell or free virus counts. It showed that, despite the adsorption efficiency $8 \mathrm{~h}$ after virus inoculation was low, with only $0.2 \%$ of infected cells, this value increased to $16 \%$ at $24 \mathrm{~h}$, and a fast lysis of the culture occurred before 48 h (Figures 2B, 3).

Another valuable application of VirusFISH was to determine the free viral particles released during infection, discriminating the true OtV5 from phages and other unspecific particles and improving the estimation of viral production in non-axenic cultures. The latter is commonly done through flow cytometry (e.g., Derelle et al., 2008; Brown and Bidle, 2014), but may have some problems due to the presence of mid-level fluorescence phages at the late stages of infection that appear with a certain overlap in the cytogram with the putative OtV5 population (Figure 5), potentially resulting in an overestimation of OtV5 viral production. We thus showed the utility of VirusFISH to detect our virus of interest within a complex viral community.

VirusFISH also unveiled that a large proportion of the viruses produced get trapped within the organic matrix released during cell lysis, which affected the quantification of the free viruses produced. It is important to note that retention of OtV5 on the $0.2 \mu \mathrm{m}$ filters will affect the same way OtV5 counts regardless of the technique used to count them (flow cytometry, plaque assay, or VirusFISH), as filtration through $0.2 \mu \mathrm{m}$ is the common procedure to remove cells and cell debris in all three techniques prior to viral counting. Thus, visualization with VirusFISH revealed a process that affects estimations of viral production and burst size values and that should be considered for future studies on viral infection dynamics.

Given the relatively low amount of OtV5 particles detected with VirusFISH, it could be argued that VirusFISH may only be counting non-encapsidated viruses. However, we find this highly unlikely. VirusFISH is applied to viral particles retained in a $0.02 \mu \mathrm{m}$ filter, whereas naked DNA would pass through the filter. Furthermore, we obtained similar counts of free OtV5 particles with flow cytometry and VirusFISH (Figure 5), and encapsidated viruses were seen surrounding cell debris in electron microscopy images (Figure 1), whereas no intermediate forms such as immature viruses were observed.

Although it was not the goal of our study due to the tiny size of Ostreococcus, VirusFISH could be potentially used for visualizing the dynamics of the viruses within the eclipse phase of infection, something that is not feasible with other methods like Transmission Electron Microscopy.

\section{Methodological Aspects to Be Considered for Phototrophic Eukaryotes and Our Particular O. tauri System}

One of the best fluorochromes to label gene probes is Alexa594 (Barrero-Canosa et al., 2017), which emits red fluorescence when excited with orange light. However, the chloroplasts of photosynthetic 
A
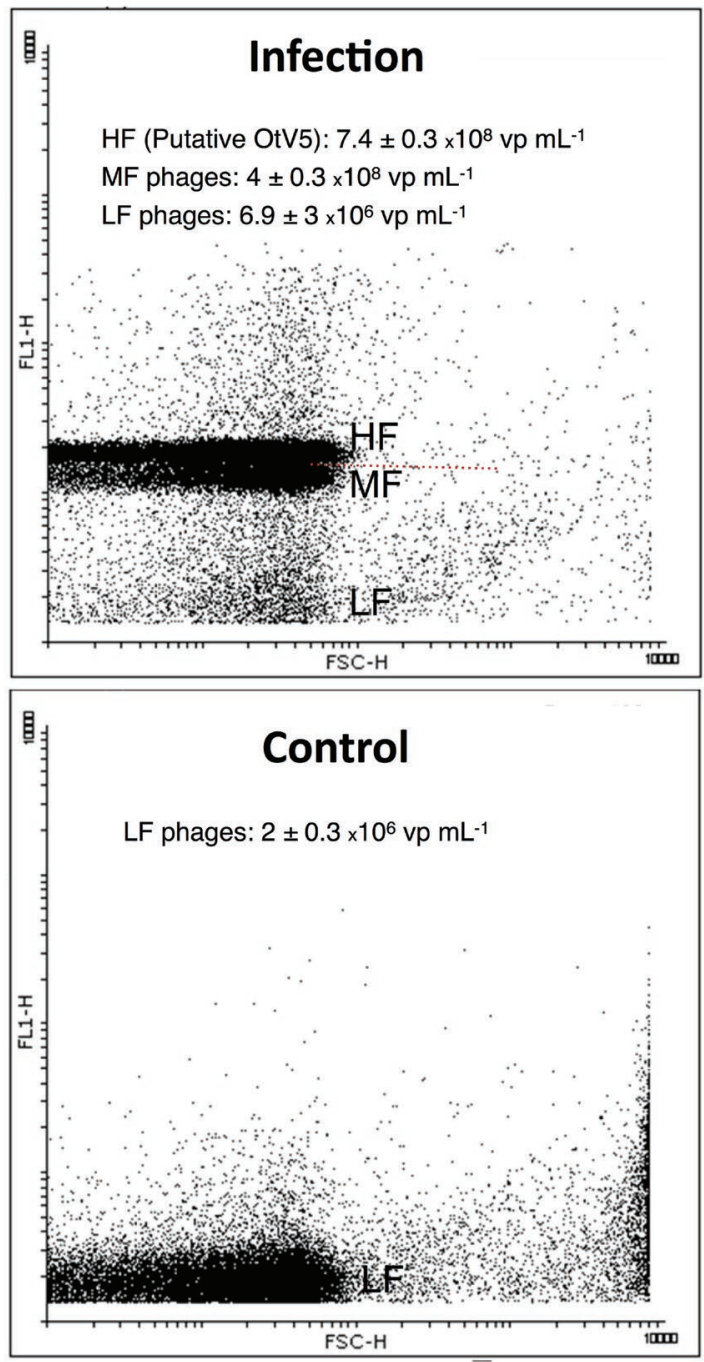

B

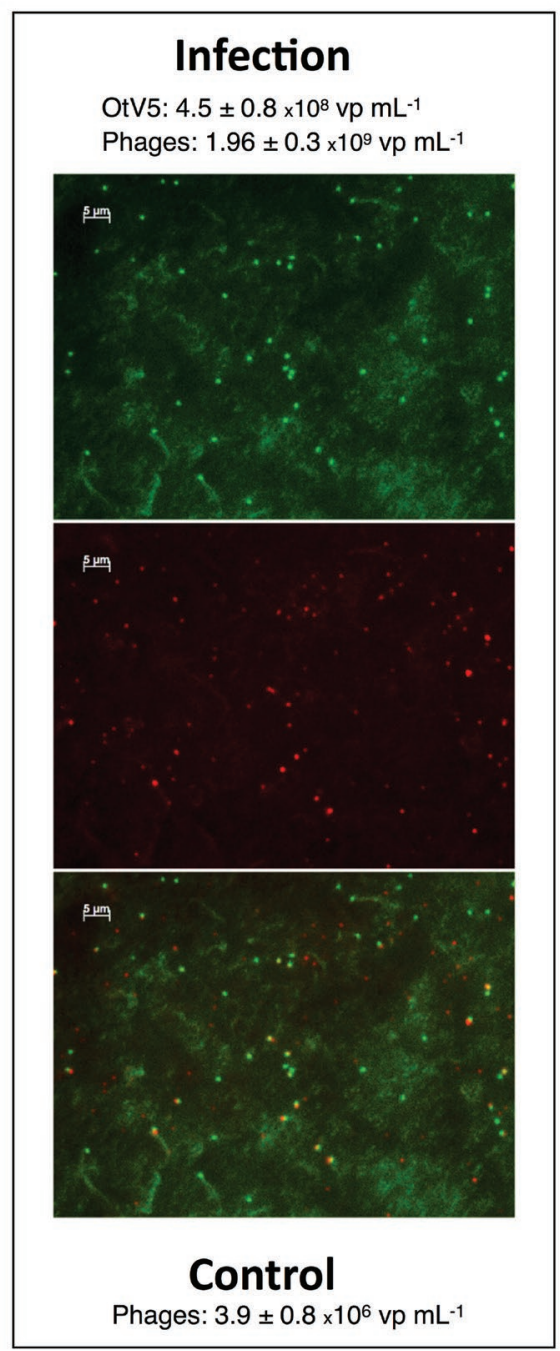

FIGURE 5 | Comparison of free virus counts using flow cytometry (FCM) and virus fluorescence in situ hybridization (VirusFISH). (A) Cytogram of viral events from an infected (96 h after OtV5 inoculation, upper panel) and a healthy O. tauri culture (Control, lower panel). The cytogram of the infected culture shows mid-level (MF) and high-level (HF) fluorescence viruses (presumably phages and putative OtV5), and also low-level fluorescence viruses (LF). The dashed red line separate MF from HF virus. Only LF viruses (phages) are seen in the control. (B) Micrographs of the $0.02 \mu \mathrm{m}$ filter of an infected culture. Top: total viruses stained with SYBR Gold. Center: VirusFISH labeled OtV5 viruses. Bottom: overlay of SYBR Gold and VirusFISH signals for OtV5 viruses. Values in the graphs represent the values obtained with VirusFISH (on the $0.02 \mu \mathrm{m}$ filter) and flow cytometry for the same $0.2 \mu \mathrm{m}$ prefiltered samples for both the infected and the healthy 0 . tauri culture.

microbes also emit red fluorescence under the same light, hampering the detection of viral signals. We solved this technical issue by removing the cellular pigments with a combination of alcohol treatments, as described in the Materials and Methods section.

The filter pore size also needs to be considered during VirusFISH experiments. Ostreococcus cells have a typical diameter of $1 \mu \mathrm{m}$, but we observed that some cells passed through $0.6 \mu \mathrm{m}$ filters, most likely because their cellular membranes are very flexible. This resulted in the loss of more than half of the cells during filtration. Consequently, we recommend the use of filters with a pore size of 0.4 or $0.2 \mu \mathrm{m}$ when working with picoeukaryotes.

\section{Modifications of VirusFISH With Respect to the Published Protocols of PhageFISH and Direct-geneFISH}

VirusFISH represents a combination between phageFISH and direct-geneFISH. It uses CARD-FISH to identify the unicellular eukaryotic host, similar to phageFISH, and uses a mixture of polynucleotide probes directly labeled with a fluorochrome to target viral genes, similar to the direct-geneFISH protocol. CARD-FISH was used because its signal amplification step enables the detection of cells with low ribosome content. O. tauri and all Mamiellophyceae have a small cytoplasm due to the relatively big size of the organelles (Henderson et al., 2007), 

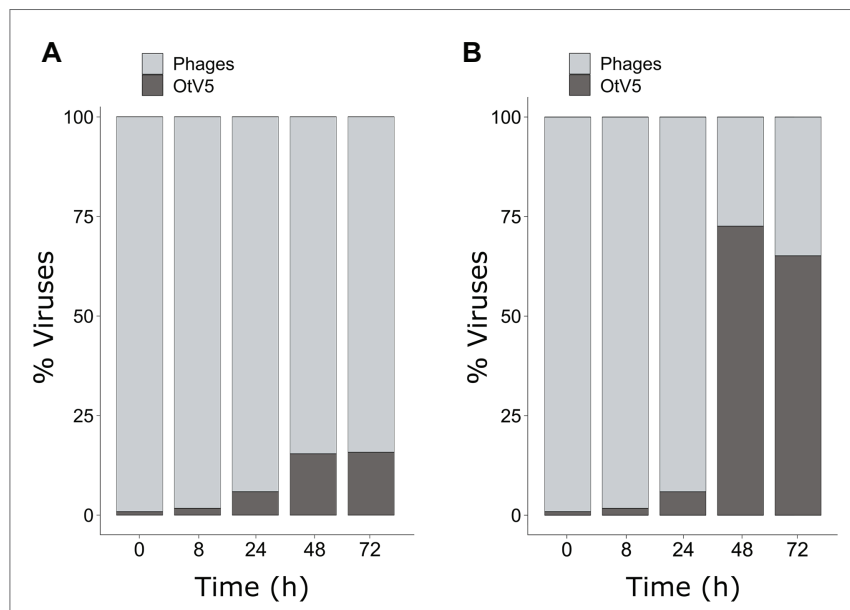

FIGURE 6 | Dynamics of the proportion of OtV5 viruses in relation to total viruses (i.e., OtV5 and phages). (A) Counts on $0.02 \mu \mathrm{m}$ filters. (B) Sum of the counts on the $0.02 \mu \mathrm{m}$ filters and the viruses retained in the cellular matrix around the cells on the $0.2 \mu \mathrm{m}$ filters. See Materials and Methods for details.

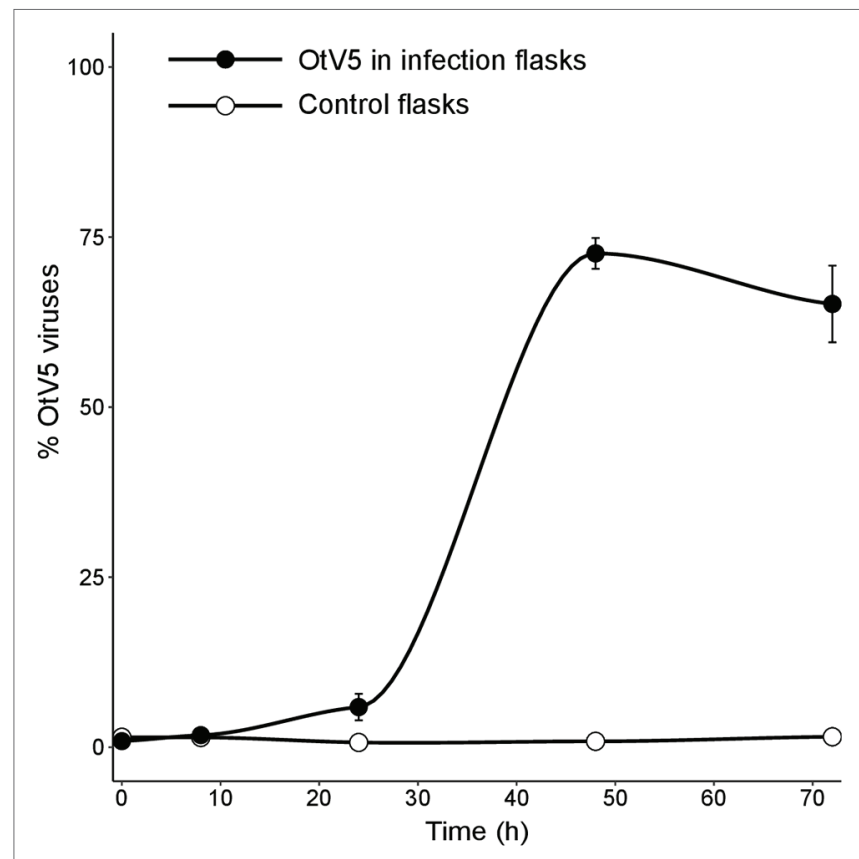

FIGURE 7 | Dynamics of free viruses produced during the infection expressed as percentage of OtV5 with respect the total viral abundance ( \pm standard error of the three replicates). Counts were done by epifluorescence microscopy considering the overlay of OtV5 and SYBR Gold signals and the retention of viruses on the $0.2 \mu \mathrm{m}$ filters at 48 and $72 \mathrm{~h}$ (see Materials and Methods and Figure 6 for details).

and therefore, their ribosomal abundance is low, and CARDFISH enhances the cellular visualization. We also incorporated a step of embedding the filters in agarose to avoid cell losses in downstream manipulations of the filter portions. Furthermore, because $O$. tauri lacks a cell wall, the permeabilization step usually needed in the CARD-FISH protocols (Pernthaler et al., 2002) was omitted. On the other hand, a treatment to completely remove cell pigments was required, as mentioned above. Finally, compared to the direct-geneFISH protocol, we reduced the Alexa594 fluorochrome volume to label the viral gene probes in order to reduce economical costs but obtaining equally optimal results (see details in the Materials and Methods section).

\section{VirusFISH vs. Other Approaches to Follow Virus-Host Dynamics}

Currently available methods to assess the dynamics between host and viruses during infection are (i) the frequency of visibly infected cells (FVIC; Wommack and Colwell, 2000), (ii) quantitative PCR (qPCR) of viral genes (Chen and Suttle, 1995; Larsen et al., 2008; Matteson et al., 2011), and (iii) the plaque assay, for counting plaque forming units (PFU; Brussaard et al., 2016). Compared with these methods, VirusFISH brings further advantages. For example, FVIC reports the fraction of infected host cells, but only detects those cells in the late stage of infection. PFU requires that the host is available in culture, and although both PFU and qPCR allow following the infection dynamics, they lack the ability to quantify the fraction of infected cells. In comparison, VirusFISH allows (i) the identification of both host and virus, using $18 \mathrm{~S}$ rRNA and specific probes that target viral genes, features particularly advantageous in non-axenic cultures of unicellular eukaryotes or in environmental samples; (ii) the quantification of the total and relative abundance of the host cells; (iii) quantification of the total and relative abundance of virus-infected cells, independent of the stage of infection; and (iv) quantification of released viral particles. Furthermore, VirusFISH could potentially be used to discriminate the different stages of infection, as it has been done with phageFISH (Allers et al., 2013).

Some other approaches have arisen in the last decade to unveil virus-host interactions, like the polony method (Baran et al., 2018) or the microfluidic digital PCR (Tadmor et al., 2011). The novel polony method is a culture independent technique based on a single molecule PCR. Using degenerate primers, it allows the determination of the abundance of a given viral group and its degree of diversity, discriminating between different viral families or genera and their host. This high-throughput approach has enabled the quantitative assessment of thousands of viruses in a single sample from both aquatic and terrestrial environments (Baran et al., 2018). Thus, the polony method is a powerful approach to detect virus-host interactions in a cost-effective and relatively simple manner, but similar to VirusFISH, it requires the knowledge of the hosts and the genome of the viral target to design the probes. However, although the VirusFISH approach is not as high-throughput as the polony method, it has the advantage that it allows monitoring viral infection dynamics, so we can see when the infection is taking place, how many cells are infected at different times, and how the infection progresses. Likewise, it allows visualizing early phases of infection or latent infections that do not result in a massive lysis of the culture.

Moreover, since VirusFISH uses microscopy observations, it enables the study of the heterogeneity of the infection within 
the host population, with the potential to extend its use to assessing specific virus-host interactions in complex natural communities.

\section{Free Viral Abundances}

The abundance of free viruses has been traditionally assessed through (i) TEM of uranyl acetate stained virus particles (Malenovska, 2013) and (ii) by epifluorescence microscopy (Hennes et al., 1995) or flow cytometry (Marie et al., 1999) of SYBR Green stained viruses. On the other hand, infective viruses are traditionally quantified by plaque assays (Suttle and Chen, 1992). Each of the above methods have limitations: (i) TEM is a time consuming, difficult to perform quantitatively, and expensive technique, (ii) SYBR staining followed by epifluorescence microscopy or flow cytometry does not distinguish between infective and non-infective viruses, and it is impossible to identify the virus of interest within a complex viral community, and (iii) the plaque-assay is constrained to cultivable hosts and their viruses. With VirusFISH we achieved the detection of specific free viruses in a relatively fast way, with no requirements of specialized equipment, or extremely expensive reagents. Allers et al. (2013) also applied phageFISH to visualize free viral particles, but they immobilized the viral lysate on glass slides, which can potentially lead to virus losses during the hybridization process. We tried to overcome this issue by collecting and counting the free viruses on $0.02 \mu \mathrm{m}$ anodisc filters decreasing the risk of viral losses during the hybridization process due to a better retention. Additionally, one filter can be used several times (by cutting the filter in portions) and kept at $-80^{\circ} \mathrm{C}$ for years. We also showed that discriminating our virus of interest from phages in non-axenic cultures is crucial for assessing viral production over time.

\section{CONCLUSION}

In summary, in this study, we developed VirusFISH to detect virus-host interaction in $O$. tauri. This technique allowed us to visualize and follow the dynamics of the OtV5 viral infection of O. tauri until the complete lysis of the culture. Also, VirusFISH enabled the calculation of the viral production during infection, discriminating OtV5 viruses from the phages present in the non-axenic culture. Our designed probes could potentially target most Ostreococcus viruses, except for OtV6, representing a valuable tool to address virus-host interactions in these cosmopolitan marine picoeukaryotes. We strongly believe that VirusFISH presents great prospects to address infection dynamics in nature, and it will foster our understanding on the impact of viruses in eukaryotic populations. Furthermore, this technique can be easily adapted and implemented on any other model system.

\section{REFERENCES}

Allers, E., Moraru, C., Duhaime, M. B., Beneze, E., Solonenko, N., Barrero-Canosa, J., et al. (2013). Single-cell and population level viral infection dynamics revealed by phageFISH, a method to visualize intracellular and free viruses. Environ. microbiol. 15, 2306-2318. doi: 10.1111/14622920.12100

\section{DATA AVAILABILITY STATEMENT}

All datasets generated for this study are included in the article/Supplementary Material.

\section{AUTHOR CONTRIBUTIONS}

YC: data curation, formal analysis, investigation, methodology, visualization, writing original draft, and review and editing. MS: data curation, formal analysis, investigation, methodology, supervision, validation, and writing - review and editing. IF: formal analysis, investigation, methodology, visualization, and writing - review and editing. NG: writing - review and editing. SY: data curation, formal analysis, and writing - review and editing. CM: methodology and writing - review and editing. DV: data curation, formal analysis, investigation, methodology, project administration, resources, supervision, validation, and writing - review and editing. All authors contributed to the article and approved the submitted version.

\section{FUNDING}

YC was supported by Ministerio de Economía, Industria y Competitividad, Gobierno de España (MINECO), grant number: BES-2014-067849 during her PhD. MS was supported by Agencia Canaria de Investigación, Innovación y Sociedad de la Información (ACIISI), grant: Viera y Clavijo 2016. Ministerio de Economía, Industria y Competitividad, Gobierno de España (MINECO), grant numbers: CTM2013-43767-P and CTM2016-75083-R supported the project and paid for the investigation (reagents, material, etc.). NG was supported by Agence Nationale de la Recherche (ANR), grant number: ANR-17-CE02-0012-03.

\section{ACKNOWLEDGMENTS}

We thank the Unitat de Criomicroscopia Electrònica (Centres Científics i Tecnològics, Universitat de Barcelona) for the electronic transmission images. This work is included as a chapter in the PhD thesis of YC (Castillo, 2019).

\section{SUPPLEMENTARY MATERIAL}

The Supplementary Material for this article can be found online at: https://www.frontiersin.org/articles/10.3389/fmicb.2020.01559/ full\#supplementary-material. diverse virus populations in the environment using the polony method. Nat. Microbiol. 3, 62-72. doi: 10.1038/s41564-017-0045-y

Barrero-Canosa, J., Moraru, C., Zeugner, L., Fuchs, B. M., and Amann, R. (2017). Direct-geneFISH: a simplified protocol for the simultaneous detection and quantification of genes and rRNA in microorganisms. Environ. Microbiol. 19, 70-82. doi: 10.1111/1462-2920.13432 
Brown, C. M., and Bidle, K. D. (2014). Attenuation of virus production at high multiplicities of infection in Aureococcus anophagefferens. Virology 466-467, 71-81. doi: 10.1016/j.virol.2014.07.02

Brussaard, C. P. D. (2004). Optimization of procedures for counting viruses by flow cytometry. Appl. Environ. Microbiol. 70, 1506-1513. doi: 10.1128/ AEM.70.3.1506-1513.2004

Brussaard, C. P. D., Baudoux, A. C., and Rodríguez-Valera, F. (2016). "Marine viruses" in The marine microbiome. eds. L. J. Stal and M. S. Cretoiu (Cham: Springer International Publishing), 155-183.

Brussaard, C. P. D., Payet, J. P., Winter, C., and Weinbauer, M. G. (2010). "Quantification of aquatic viruses by flow cytometry" in Manual of aquatic viral ecology. eds. S. W. Wilhelm, M. G. Weinbauer, and C. A. Suttle (Waco, TX: American Society of Limnology and Oceanography, Inc.), 102-109.

Cabello, A. M., Latasa, M., Forn, I., Morán, X. A. G., and Massana, R. (2016). Vertical distribution of major photosynthetic picoeukaryotic groups in stratified marine waters. Environ. Microbiol. 18, 1578-1590. doi: 10.1111/1462-2920.13285

Castillo, Y. M. (2019). Interactions between marine picoeukaryotes and their viruses one cell at a time. dissertation thesis. Barcelona (SP): Institute of Marine Sciences-CSIC.

Castillo, Y. M., Mangot, J., Benites, L. F., Logares, R., Kuronishi, M., Ogata, H., et al. (2019). Assessing the viral content of uncultured picoeukaryotes in the global-ocean by single cell genomics. Mol. Ecol. 28, 4272-4289. doi: 10.1111/mec.15210

Chen, F., and Suttle, C. A. (1995). Amplification of DNA polymerase gene fragments from viruses infecting microalgae. Appl. Environ. Microbiol. 61, 1274-1278. doi: 10.1128/AEM.61.4.1274-1278.1995

Chow, C. E. T., Winget, D. M., White, R. A., Hallam, S. J., and Suttle, C. A. (2015). Combining genomic sequencing methods to explore viral diversity and reveal potential virus-host interactions. Front. Microbiol. 6:265. doi: $10.3389 /$ fmicb.2015.00265

Chrétiennot-Dinet, M. -J., Courties, C., Vaquer, A., Neveux, J., Claustre, H., Lautier, J., et al. (1995). A new marine picoeucaryote: Ostreococcus tauri gen. et sp. nov. (Chlorophyta, Prasinophyceae). Phycologia 34, 285-292. doi: $10.2216 / 10031-8884-34-4-285.1$

Clerissi, C., Desdevises, Y., and Grimsley, N. (2012). Prasinoviruses of the marine green alga Ostreococcus tauri are mainly species specific. J. Virol. 86, 4611-4619. doi: 10.1128/JVI.07221-11

Countway, P. D., and Caron, D. A. (2006). Abundance and distribution of Ostreococcus sp. in the San Pedro Channel, California, as revealed by quantitative PCR. Appl. Environ. Microbiol. 72, 2496-2506. doi: 10.1128/ AEM.72.4.2496-2506.2006

Derelle, E., Ferraz, C., Escande, M. -L., Eychenié, S., Cooke, R., Piganeau, G., et al. (2008). Life-cycle and genome of OtV5, a large DNA virus of the pelagic marine unicellular green alga Ostreococcus tauri. PLoS One 3:e2250. doi: 10.1371/journal.pone.0002250

Guillard, R. R. L., and Hargraves, P. E. (1993). Stichochrysis immobilis is a diatom, not a chrysophyte. Phycologia 32, 234-236.

Guillou, L., Eikrem, W., Chrétiennot-Dinet, M. -J., Le Gall, F., Massana, R., Romari, K., et al. (2004). Diversity of picoplanktonic prasinophytes assessed by direct nuclear SSU rDNA sequencing of environmental samples and novel isolates retrieved from oceanic and coastal marine ecosystems. Protist 155, 193-214. doi: 10.1078/143446104774199592

Heath, S. E., and Collins, S. (2016). Mode of resistance to viral lysis affects host growth across multiple environments in the marine picoeukaryote Ostreococcus tauri. Environ. Microbiol. 18, 4628-4639. doi: 10.1111/1462-2920.13586

Henderson, G. P., Gan, L., and Jensen, G. J. (2007). 3-D ultrastructure of O. tauri: electron cryotomography of an entire eukaryotic cell. PLoS One 2:e749. doi: 10.1371/journal.pone.0000749

Hennes, K. P., Suttle, C. A., and Chan, A. M. (1995). Fluorescently labeled virus probes show that natural virus populations can control the structure of marine microbial communities. Appl. Environ. Microbiol. 61, 3623-3627. doi: 10.1128/AEM.61.10.3623-3627.1995

Labonté, J. M., Swan, B. K., Poulos, B., Luo, H., Koren, S., Hallam, S. J., et al. (2015). Single-cell genomics-based analysis of virus-host interactions in marine surface bacterioplankton. ISME J. 9, 2386-2399. doi: 10.1038/ismej.2015.48

Larsen, J. B., Larsen, A., Bratbak, G., and Sandaa, R. -A. (2008). Phylogenetic analysis of members of the Phycodnaviridae virus family, using amplified fragments of the major capsid protein gene. Appl. Environ. Microbiol. 74, 3048-3057. doi: 10.1128/AEM.02548-07
Malenovska, H. (2013). Virus quantitation by transmission electron microscopy, TCID50, and the role of timing virus harvesting: a case study of three animal viruses. J. Virol. Methods 191, 136-140. doi: 10.1016/j.jviromet.2013.04.008

Marie, D., Brussaard, C. P. D., Thyrhaug, R., Bratbak, G., and Vaulot, D. (1999). Enumeration of marine viruses in culture and natural samples by flow cytometry. Appl. Environ. Microbiol. 65, 45-52. doi: 10.1128/AEM.65.1.45-52.1999

Massana, R. (2011). Eukaryotic picoplankton in surface oceans. Annu. Rev. Microbiol. 65, 91-110. doi: 10.1146/annurev-micro-090110-102903

Matteson, A. R., Loar, S. N., Bourbonniere, R. A., and Wilhelm, S. W. (2011). Molecular enumeration of an ecologically important cyanophage in a laurentian Great Lake. Appl. Environ. Microbiol. 77, 6772-6779. doi: 10.1128/AEM.05879-11

Monier, A., Chambouvet, A., Milner, D. S., Attah, V., Terrado, R., Lovejoy, C., et al. (2017). Host-derived viral transporter protein for nitrogen uptake in infected marine phytoplankton. Proc. Natl. Acad. Sci. 114, E7489-E7498. doi: 10.1073 /pnas.1708097114

Noble, R., and Fuhrman, J. (1998). Use of SYBR Green I for rapid epifluorescence counts of marine viruses and bacteria. Aquat. Microb. Ecol. 14, 113-118. doi: $10.3354 / \mathrm{ame} 014113$

Not, F., Latasa, M., Marie, D., Cariou, T., Vaulot, D., and Simon, N. (2004). A single species, Micromonas pusilla (Prasinophyceae), dominates the eukaryotic picoplankton in the Western English Channel. Appl. Environ. Microbiol. 70, 4064-4072. doi: 10.1128/AEM.70.7.4064-4072.2004

Pavlekovic, M., Schmid, M. C., Schmider-Poignee, N., Spring, S., Pilhofer, M., Gaul, T., et al. (2009). Optimization of three FISH procedures for in situ detection of anaerobic ammonium oxidizing bacteria in biological wastewater treatment. J. Microbiol. Methods 78, 119-126. doi: 10.1016/j.mimet.2009.04.003

Pernice, M. C., Forn, I., Gomes, A., Lara, E., Alonso-Sáez, L., Arrieta, J. M., et al. (2015). Global abundance of planktonic heterotrophic protists in the deep ocean. ISME J. 9, 782-792. doi: 10.1038/ismej.2014.168

Pernthaler, A., Pernthaler, J., and Amann, R. (2002). Fluorescence in situ hybridization and catalyzed reporter deposition for the identification of marine bacteria. Appl. Environ. Microbiol. 68, 3094-3101. doi: 10.1128/ AEM.68.6.3094-3101.2002

Porter, K. G., and Feig, Y. S. (1980). The use of DAPI for identifying and counting aquatic microflora. Limnol. Oceanogr. 25, 943-948. doi: 10.4319/ lo.1980.25.5.0943

Suttle, C. A., and Chen, F. (1992). Mechanisms and rates of decay of marine viruses in seawater. Appl. Environ. Microbiol. 58, 3721-3729. doi: 10.1128/ AEM.58.11.3721-3729.1992

Tadmor, A. D., Ottesen, E. A., Leadbetter, J. R., and Phillips, R. (2011). Probing individual environmental bacteria for viruses by using microfluidic digital PCR. Science 333, 58-62. doi: 10.1126/science.1200758

Thomas, R. (2011). Action des phycoDNAvirus sur les populations phytoplanctoniques (Mamiellophyceae): étude de la résistance aux infections virales. $\mathrm{PhD}$ thesis. France: Université Pierre et Marie Curie.

Weynberg, K. D., Allen, M. J., Gilg, I. C., Scanlan, D. J., and Wilson, W. H. (2011). Genome sequence of Ostreococcus tauri virus OtV-2 throws light on the role of picoeukaryote niche separation in the ocean. J. Virol. 85, 4520-4529. doi: 10.1128/JVI.02131-10

Weynberg, K., Allen, M., and Wilson, W. (2017). Marine prasinoviruses and their tiny plankton hosts: a review. Viruses 9:43. doi: 10.3390/v9030043

Wommack, K. E., and Colwell, R. R. (2000). Virioplankton: viruses in aquatic ecosystems. Microbiol. Mol. Biol. Rev. 64, 69-114. doi: 10.1128/MMBR.64.1.69114.2000

Yau, S., Hemon, C., Derelle, E., Moreau, H., Piganeau, G., and Grimsley, N. (2016). A viral immunity chromosome in the marine picoeukaryote, Ostreococcus tauri. PLoS Pathog. 12:e1005965. doi: 10.1371/journal.ppat.1005965

Conflict of Interest: The authors declare that the research was conducted in the absence of any commercial or financial relationships that could be construed as a potential conflict of interest.

Copyright (ㅇ 2020 Castillo, Sebastián, Forn, Grimsley, Yau, Moraru and Vaqué. This is an open-access article distributed under the terms of the Creative Commons Attribution License (CC BY). The use, distribution or reproduction in other forums is permitted, provided the original author(s) and the copyright owner(s) are credited and that the original publication in this journal is cited, in accordance with accepted academic practice. No use, distribution or reproduction is permitted which does not comply with these terms. 